I. TNTRODUCTION . . . . . . . . . . . . . . 1

A. Computer Environment ............. 2

B. Availability of Programs ........... 3

II. Electric and Magnetic Field Programs

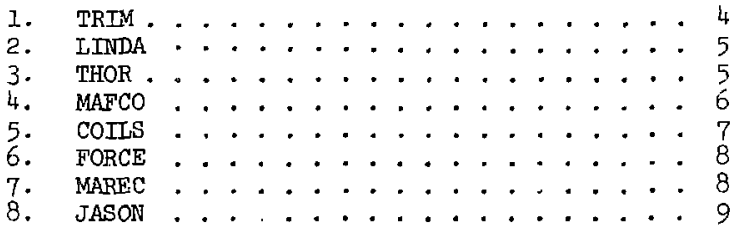

III. Computer Programs for Orbit Calculetions

A. Using Matrix Formalism

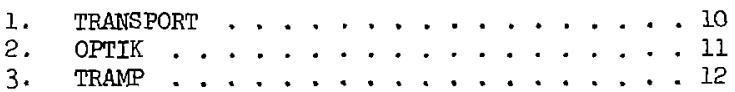

B. Using Integration Techniques

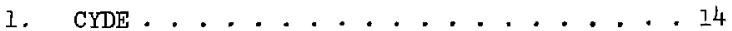

2. $\operatorname{GOC} 3 \mathrm{D} \cdot . \cdot . \cdot . \cdot . \cdot . \cdot . \cdot . \cdot .+16$

3. TRAJECTORY . . . . . . . . . . . . . 17

4. SOLRM . . . . . . . . . . . . . . . 18

5. PINWHEEL . . . . . . . . . . . . . . . 18

6. SYNCH ....................... 19

7. MAGOP ... . . . . . .......... 20

IV. Computer Programs for Circuit Design

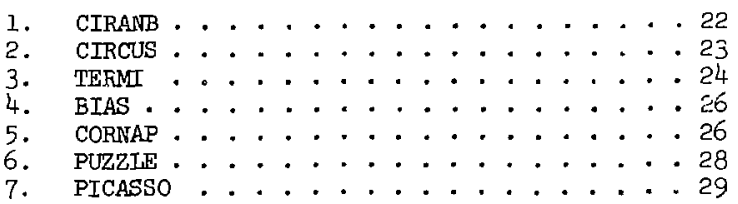

AFPENDICES

Appendix I. Bibliography . . . . . . . . . . 30

Appendix II. Program Summary Form . . . . . . 35

\title{
NOTICE
}

This report was preparad as an account of work oporsorec by the Inited Stutes Government. Nolther the United Stakes nos the Unleed States Atcinic Ensrgy Commledon, nor any of their empioyees, nor any of thet contractors, subcontractors, or th: I employaes, makes eny warraty, exprese or inplled, of asumes any Iegal linbility or responsiblity for the eccurucy, compietexass or urefuinces of any informatian, apparatus, product or process disclosed, or cepresents thet ite $d \mathrm{~b}$ would not infringe privately owned rights. 
COMPUTER PROGRAMS FOR

ACCELERATORS AND ELEC TRONIC CIRCUIT DESIGNS

\author{
John S. Colonias \\ Lawrence Berkeley Laboratory \\ University of California \\ Berkeley, California
}

December, 1971

\title{
ABSTRACT
}

This is the first of a series of publications intenden to assemble under one cover the most important computer programs relative to various major activities in this Laboratory, and thus establish a permanent library of such programs. This publication will deal specifically with computer programs relating to accelerator design and electronic circuit design. Each program is described in terms of an abstract, and a table of vital statistics summarizes the most important information relative to that program. 
INTRODUCTION

The purpose of this publication is twofold: first to assemble under one cover descriptions of the most important and commonly used computer programs for accelerator design and circuit design, and secondly to establish a permanent library of such programs.

The necessity of such a library of programs stems from the magnitude and complexity of the problems involved, as well as from the large capital investment required to develop and maintain such computer programs. In the past, duplication of effort and the consequent unnecessary expense, has created a large number of computer programs whose function in most respects is similar--not to say identical.

Most of the programs herein described have been utilized effectively by other laboratories. They are general in concept, easy to implement in computer environments other than the ones cited, they are fully documented and operational.

The subject matter of this document will be published by Academic Press in more extensive form in a frorthcoming book entitled "Gomputer Programs fcr Accelerator Design".

In this report an abstract of each program is given elong with a table of vital statistics, so that the potential user may detrrmine immediately whether the program meets his requirements.

It is anticipated that this report will. be updated periodically, to reflect adaitional programs developed, or contributed from elsewhere and revisions to existing codes.

Perhaps, the rosi important information contained in this report, relates to the fact that some of the programs listed herezin have been modified, to run "on-line" from remote installations, through a communications terrinal. 
Eventually all programs listed will become available in this mode of operation so that users from other installations may avail themselves of this service.

\section{Computer Environment}

To familiarici the potential user with the computing facility at IRI, Berkeley, the following birds-eye-view is given.

Presently, there are two CDC 6600 and one CDC 7600 computer systems at Irawrence Radiation Laboratory in Berkeley, running respec.. tively BKY and SCOPE operating systems.

For remote and interactive prucessing the Laboratory has implemented a system which allows a large number of users to utilize the computer facilities from remote locations. Approximately 120 teletypes channels are available for this purpose.

For semi-permanent data storage, there is an IBM data cell and an IBM 1360 photodigital storage system (the chipstore). Both systems are on line to all Laboratory computer. The IBM 1360 is capable of storing a third of a trillion bits of data which is equivalent to almost 3000 full magnetic tape.

To perform interactive and remote functions software packages have been developed which allow the user to perform the tasks which are listed below:

1. EDIT This facility provides for entering text on-line into a buffer, and for subsequently examining and modifying part or all of that text. 
2. COMUT This subsystem evaluates a one line arithn心tic replacement statement. This subsystem allows the terminal to be used as a sophisticated desk calculator,

3. IOAD This subsystem permits the loading of a program stored in semi-permanent storage.

4. STORE Permits the storing of a generated program (file):

5. $\mathrm{XEQ}$ This subsystem consists of an interpreter for a subset of FORTRAN IV. Its function is to execute programs entered via the text editor (EDIT) subsystem.

6. SUBMIT This subsystem permits submission of a program into the normal batch system.

Remote batch service will be available on about August 1, 1971 : to users of the Laboratory's compuiving facility.

\section{Availability of Programs}

The programs listed in this publication are available from the LRT Computer Iibrary, if interested write to:

Miss Judy Lawrence

Computer Library

Building 50B, Room 1232B

Lawrence Radiation Laboratory

Berkeley, California 94720

Questions relating to problem definition or to program operation should be aãdressed to:

John S. Colonias

Mathematics and Computing

Building 50B, Room 3209

Lawrence Radiation Laboratory

Berkeley, California 94720 
For further information contact:

$$
\begin{aligned}
& \text { Mr. Eric Beals } \\
& \text { Head, Users Services Group } \\
& \text { Msthematics and Computing } \\
& \text { Lawrence Berkeley Laboratory } \\
& \text { Berkeloy, CA } 94720
\end{aligned}
$$

\section{Availability of Programs}

The programs listed in this publicaton are available from the LBL Computer Library; if interested write to:

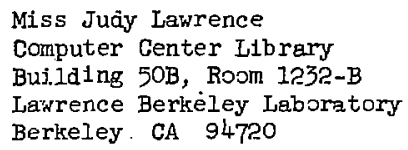

Wuestions relating to problem definition or to program operation should be addressed to:

John S. Colonias

Mathematics and computing

Building 50B, Room 3209

Lawrence Berkeley Laboratory

Berkeley, CA 94720 
 \\ EIECTRIC and MAGNETIC FIELD PROGRAMS}

1. TRIM $^{1,2}$

This program has been extensively used for the design of accelerator magnets. It is a general purpose, two-dimensional magnetostatic code capable of solving mathematical models of two dimensional magnets. It includes the effecis of finite nonuniform permeability of the iron. The universe of the problem is composed of irregular interfaces and boundaries.

Additional features include:

a. Variable triangulax mesh of about 4000 points

b. No geometrical restrictions are imposed. Any shape of magnet may be considered

c. Symmetry about the median plane is not required, therefore, both symmetric and asymmetric magnets may be considered

d. Any current distribution or conductor orientation may be considered

e. Axially symmetric magnets may also be considered

f. Energy stored in air and iron regions is calculated

g. Boundary value problems may be considered

h. Constant $\left(Y=\frac{I}{\mu}\right)$ may also be solved.

Auxiliary programs allow the Calcomp or CRT plotting of the resulting flux distribution and of the printing tables of the components of the magnetic field throughout the universe of the program.

A special interactive teletype version of this program exists allowing the user to submit and execute from a remote station. 
2. IINDA $^{3}$

Program LINDA uses a combination of scalar and vector potential to rociel two-dimensional magnetostatic problems with a single general interface, and iron of finite non-uniform permeability. The computer model consists of a rectanguiar problem space or wiverse with a single region of iron and one region (sometines two) of air. There may be rnany current conductors inside or outside the problem space but currents in iron are not allowed. The problem space is divided into a mesh of uniform rectangles in which the magnet geometry is outlined. The program does not allow separate pieces of iron, however, it provides for a very flexible urray of topologically defined types of magnets which provide the necessary information about the location of the interface on mesh borders.

LINDA is a very fast program, and it provides accurate results Auxiliary programs allow for CalComp plotting of the resulting flur distribution and the printing of special tables of field components, permeabilities etc. at the discretion of the user

3. $\mathrm{THOR}^{4}$

This is a general purpose three-dimensional magnetostatic program based on the one potential (vector) throughout. The algorithm utilizes a variational principle for the nonlinear magnetostatic field problem. A finite-element approximation for an arbitrary mesh is constructed from this principle, and the resulting equations are solved by nonlinear successive overrelaxation. 
The program follows the same pattern as TRIM, that is it consists of a problem GENERATOR which uses a variable triangular prism mesh on which the magnet geometry is outlined. The GENERATOR calculates various quantities dependent on the magnet configuration and prepares a magnetic tape which is read by the second part of the program responsible for the calculation of the resulting magnetic fields.

The input definition is relatively simple and follows the same procedure as TRIM.

Presently, THOR, has produced acceptable solutions, with no convergence problems, however, the rate of convergence has been slow and means to increase its speed are being investigated.

The accuracy of the program is also being tested by comparing the calculated field components with those of measured fields.

4. MAFCO $^{5}$

This computer program is capable of calculating the magnetic fields resulting irom a given set of current-carrying conductors of arbitrary two or three dimensional geometry. The code is particularly well adopted to complicated geometries arising in plasme physics, however, it may be used in other instances where a generalized two or three dimensional solution is desired for a coil geometry in which no permeable material is present. The elements which compose the generalized coil geometry are approximated by:

a. Circular loops with designeted position and orientation in space. 
b. Circular arcs with designated position and orientation space.

c. Helices along the $z$-axis (in the cylindricel coordinate system) with any designated pitch, starting point and ending point.

d. Straight lines with arbitrary orientation.

e. General elements specified by a list of points which the program connects with straight lines.

The program provides for flexible output of the components of the fields at specified grid points, or CALCOMP plots of field lines and isobars of constant $|B|$.

5. $\operatorname{coIIs}^{6}$

COIIS is a general purpose computer program to calculate vector potentials, axial and radial components of magnetic fields, forces and mutual inductances between coaxial conductors or to plot lines of force, lines of constant $B, B_{2}$ etc., of any system composed of current carrying elements.

These elements may be any combination of circular filaments, thin solenoids, cylindrical or plane current sheets, or thick cylindrical coils with rectengular cross sections. The computed fields are accurate even within the windings of the coils, and in most cases the accuracy is to a minimum of seven significant digits.

This program has been adepted to run interactively through a teletype terminal, allowing the user to exercise control over the operation of the program. The user may alter, delete, or 
add conductors, print selective output and return to the computational loop for more calculations based on modifled data.

6. FORCE 7

This progrem is an extension of the previously described program MAFCO, and it is used to calculate the magnitude and direction of the local magnetic force.

Once the magnetic field resulting from the coll configuration specified has been calculated, FORCE, using Fouraboy's law. calculates the magnetic force at the center of each straight line section according to the order in which these appear in the input data.

Since FORCE calculates forces for only straight line section, it is imperative to approximate curvilinear conductors by a serles of straight line segments. Obviously the length of eech line segment will determine the degree of refinement of the resulting force distribution, thus providing a handle for coarse or fine calculetions at the 3xpense or saving of computer time. 7. MAREC $^{8}$

This program computes two-dimensional static magnetic fields and includes the effects of finite permeability of the iron. MAREC makes use of the modified scalar potential (same principal as program LINDA). The problem universe is divided in two parts: the air-coil region and the iron region. These parts are computed separately, thus realizing a considerable saving of computer time and memory space. MAREC was developed at CERN and has been used extensively in the magnet design studies of the intersecting storage ring profect and in the present studies for the new 300-500 GEV facility. 
The program is general enough to accept any reasonable pole configuration, including concavities, several minima, and a large number of coils of any shape.

The computer model consists of a rectangular mesh of 3250 points for the air region and 2900 points for the iron region. 8. JASON $^{8}$

This is a two-dimensional ej.ectrostatic program capable of solving a variety of problems encountered in the design of electrostatic lenses, deflecting system, and in general, problems relating to the static evaluation of electric fields. Some of the outstanding characteristics of the program include: 1) use of both cylindrically symmetric systems and two-dimensional cartesian systems, 2) completely general boundary conditions (Neumann, Dirichlet), 3) generalized quadrilateral mesh, 4) use of block interactive methods for the solution of the equations, 5) ease and simplicity of input, 6) consideration of nonhomogenous anisotropic media, 7) flexible output and Calcomp plots of fields and equipotential lines. 


\section{COMPUTER PROGRAMS FOR ORBIT CALCULATIONS}

\section{A. Using Matrix Formalism}

1. TRANSPORT ${ }^{10,11}$

Perhaps the most versatile and widely applicable program for the design of beam transport systems is the program TRANSPORT. This program was originally written in the BALGOL language, and has subsequently been translated to FORTRAN. The version at LRL has the features listed below:

a. Flexible array of beam elements

b. First-and second-order optics

c. Tracking up to 50 vectors $\left(x, x^{\prime}, y, y^{\prime}, \delta s, \frac{d p}{p}, p\right)$

d. Transformation of apertures forward and backward in phase space to create acceptance polygons. The calculations may be cycled over energy so as to obtain energy spectra.

e. Plotting on output peper the beam ellipsoid projections and the locations of up to 50 vectors in any phase plane desired.

f. Plotting on output paper (at the end of each run) the beam line showing ellipse projections, vector projections and apertures.

g. Space-charge calculation

h. Calculations of Betatron functions

i. Separators may be included in the beam line. TRANSPORT helps the beam designer by providing him with a flexible array of beam elements of which to develop his beam line. Many of the parameters may be varied subject to specified 
constraints on the beam ellipsoid (or transformation matrix). Problems involving unknown quadrupole excitaticas, spacings' etc. may be solved.

Three main versions of TRANSPORT exist at IRL

1. off-line version (standard version)

2. On-line version. This version of TRANSPORT uses the CDC 252 displey console. Existing software provide for the utilization of the light pen, for programmable interrupts, use of teletype to enter or extract data and for performing various on-line plotting functions. This interactive mode of operation has increased the the usefulness of TRANSPORT, by allowing the user to become a dynamic part of the computational loop.

3. Teletype version. The same interactive features or (2) above, but without the display console. This version may run remotely through the BRF system, providing the user the same interactive features and flexibility.

Selective teletype printout options are available and free format input routines add to the flexibility of the program.

2. OPTIK $^{12,13}$

This program as well as TRANSPORT, provide the experimenter with the facility to handle problems encountered in the optical design of high energy particie beams. It was originally written by Devlin for the IBM 709 Computer System, and it has been modified considerably by Chaffee and others to increase its usefulness and flexibility. 
The present version of OPTIK, allows the experimenter to track particles through a beam transport system consisting of

\section{a. Field free regions}

b. Bending magnets (either wedge-shaped or rectangular regions of homogeneous magnetic field)

c. Quadrupole lenses with one or two degrees of freedom

d. Velocity separators.

The program produces solutions only to first order calculation for displacement and divergence, and first and second order for momentum dispersion. Various forms of output are avajiable as well as Calcomp plots of phase space area and ray tracing plots. One severe limitation of the program is that in satisfying a given condition OPIIK will vary only the strength of the quadrupole Ienses. Another restriction is that no more than two variable symmetric triplet can be treated in any one jeam section.

3. TRAMP $^{14,15}$

Program TRAMP was developed at Rutherford High Energy Laboratory by Gardner and Whiteside to provide solutions to problems encountered in beam transport design. It has been extensively modified by various experimenters in the field to fit the needs and the computer environments of their respective Laboratories.

The version mentioned here is capable of tracking and matching trajectories, beam profiles, or phase space ellipses through a given beam transport system. The beam elements that may be used in TRAMP are represented by $2 \times 2$ matrices for each 
plane, transforming the displacement-divergence vectors from beginning to end.

Matching beam condtions are achieved by breaking up the beam into sections, and by maksing systematic changes in specified variable elements, setting up matrices for each section, uniil, through an iterative procedure, the product mutrix is determined corform with the specified matching concitions. The program is capable of matching for: a) focal and phase-pace conditions, b) wajst and/or magnification matching and c) matching of dispersion and separation conditions.

A special version of TRAMP allows the combination of OPTIK and TRAMP plus graphic display interaction routines, thus providing on-line capabilities. 
B. With Integration Techniques

1. $\mathrm{CYDE}^{16}$

This is a collection of cyclotron development programs used to calculate:

a. Isochronous equilibrium orbit properties

b. Predict trim coil currents which give a least squares fit to a desired magnetic field shape

c. Magnetic fields produced by sets of trim coil currents. These are clurrents used in previous cycjotron runs, and also currents predicted by the least squares fit program.

d. Phese history, SINO, for both previously run beams and least squares predicted

e. Fourier coefficients for a harmonic analysis of measured field data.

Specifically, the programs responsible for these functions are:

a. LESCO. Obtains a base megnetic field $B(I, R, \theta)$ for use in the rest of the codes. Thus, given a current I and an input tape containing interpolating polynomial coefficiert IESCO constructs an output tape containing $R(I, R, \theta)$.

b. DORO. This progrem is mainly concerned with the calcillation of an isochronous field $B_{s}(R, \theta)$, and as a byproduct it furnishes orbit properties corresponding 
to the specified field (e.g., $v_{z}, v_{r}$ ). If desired IORO will Fourier analyze the fields.

c. CYDEB. It furnishes field effects $d B / d I$ for the main field and trim coils, and calculates vartous field derivatives. These quantities will be used either in DOR88 (a subroutine of CYDEA) or in TRTMCO.

When called DOR88 takes specified coil currents as input and using these values obtains a constructed field $B=B(I, R, \theta)+\sum_{J}\left(\frac{d B}{d I}\right)_{J} \Delta I_{J}$, the sine of the phase, SINO(R)-SINO 0 and various associated field derivatives.

d. TRIMCO. This is the least square fitting routine. The procedure used is based on specifying a desired average magnetic field vs radius. This is obtained on $a$ "model beam" by running various field shapes and selecting the "model beam" it is used to predict all the other particles and energies in the same scaling mode, i.e., with the same number of particle revolutions during acceleration. For example, the model beam of $50 \mathrm{MeV}$ -particle with $50 \mathrm{KV}$ on the dee, has the same number of revolutions on $60 \mathrm{MeV}$ or at $60 \mathrm{kV}$ on the dee, or $30 \mathrm{MeV}$ protons with 60KV on the dee ete. The field profile for TRIMCO is given relative to the isochronous field, or as the required field divided by the isochronous field. 
2. ${\operatorname{GOC} 31^{17,11}}^{11}$

This is generel orbit code incorporating a flexible selection of magnetic field input geometrias ${ }^{2}$ encountered in accelerator design. The code uses a three dimensional field array or a two dimensional field map, if median plane symmetry is assumed. It will track up to 60 specified particles, truck a grid of phase space, or calculate equilibrium orbit properties using the methods of Gordon and Walton. The code also provides for the calculation of the first order transfer matrix for small deviations about any trajectory.

Theta $(\theta)$, is taken as the independent variable and the equations for $r, p_{r}, z, p_{z}$, and $T$ (time) are simultaneously integrated. The energy may be changed during the integration process, by action of R. F. acceleration or a scaling of $E$ and $B$ to simulate Betatron acceleration.

Orbit output is available at every integration step, every revolution, or every $n^{\text {th }}$ revolution. Additional orbit output may be requested at up to four spectal azimuths which lie on Integration steps. The code has been used for the study of isochronous cyclotrons, the 184 j.nch synchro-cyclotron, the electron ring accelerator project and the $5 \mathrm{MeV}$ Livermore storage ring.

Two versions of this cade are now avallable,

1) AGOC3D with 3000 points in the main and perturbation fleld arrey

2) 3GOC3D with 3000 points in the main and 33000 points in the perturbation fleld map. 
3. TRAJECTORY 18

This program integrates simultaneously the equations of motion of a charged particle in a magnetic field and the differential equations for the ion optic matrix representing small motion about the paraxial trajectory. The orbit calculation is carried out in the median plane with the vertical transformation matrix calculated from a Taylor series expansion of the magnetic fleld off the median plane. The matrix so calculated is readily used with the iron-optic trensport codes TRANSPORT or OPIIK. The trajectory data can be used to obtain internal target positioning for a given bear line.

The charged particles (protons, positive or negative pions, etc.) can be started individually from a specified $\mathrm{R}, \theta$ and $\$$ and tracked forwerd to find the trajectory treced by a particle leaving a traget, or can be run backward so that the beam line can be extended back into the cyclotron to find the required target location. The particles can, also, be started from a rectangular array of $R, \phi,(P r)$ values at a fixed azimuth $\theta$ so that a slit or aperture acceptance can be determined by reverse tracking.

The orbit calculation can be stopped at any desired radius, azimuth, or value of trajectory length, so that the accuracy of the matrix can be checked by comparison with the matrix determind by actual ray tracing.

Detailed output is provided with the trajectory printed in polar and rectangular coordinates. 
4. SOTRM $^{19,20}$

This program generates first and second order transformation elements by integrating numerically through a specified magnetic fjeld a specific set of rays. SOTRM can also ray trace up to 30 nearby rays while integrating for the central orbit. For the purpose of evaluating the signifleance on the generated elements, it has the ability to apply a previously generated set of elements to the initial conditions of the nearby rays currently being obtained by integration and thus compare the integrated values of the solution with those obtained by applying the trensformation.

This program consists of a number of subrouilines and it is easily adaptable to verious physical problem.

5. PINWHEEL 21,22

This program traces trajectories of charged particles moving in a combined electric and magnetic field. The problem considered is

$$
\frac{d^{2} q}{d t^{2}}=\left(\frac{q}{m}\right)\left(E+\frac{\partial r}{\partial t}+B\right)
$$

where $r=(x, y)$ is the radius vector, E=-grad $v: 3$ the electric field and $B$ is the magnetic flux density. The initiel conditions are:

$$
\begin{aligned}
& \frac{\partial r}{\partial t}(0)=r_{0} \\
& r(0)=r_{0}
\end{aligned}
$$


This program is a modification of the Michigan State University version of PINWHEEL and as it is presently written it is restricted to two-dimensional cases with $E=\left(\mathrm{E}_{\mathrm{xl}} \mathrm{E}_{\mathrm{v}}\right)$ and $\mathrm{B}=\mathrm{B}_{\mathrm{z}}$. It integrates $\mathrm{Eq} .(1)$ and tabulates the position $(\mathrm{x}, \mathrm{y})$, the momentum $\left(\mathrm{p}_{\mathrm{x}}, \mathrm{p}_{\mathrm{y}}\right)$ and the kinetic energy versus the integration variable time. If desired, orbit center points may be output and orbits may be plotted using a Calcomp plotter.

This program is useful for orbit tracking, and was developed for cyclotron center region studies.

6. SYNCH $^{23}$

This program obtains certain information relative to transverse or bit motion in a synchrotron, such as calculation of the betatron function, the momentun-compaction, and the closed orbit in the presence of misalignments. At present, the code solves problems for which the accelerator may be thought of as made up of the following linear elementa:

1. Drift spaces

2. Bending magnets with uniform gradient

3. Quadrupole magnets

4. Misalignments

5. Arbitrary elements speciflable by input matrices

6. Other transformations not expressible as matrices

For each element the corle constructs two $3 \times 3$ matrices respectively describing the transverse motion in the radial and vertical planes. These matrices are to be thought of as operatins respectively either on the column vectors $\left(x, x^{\prime}, \Delta p / p\right)$ and $\left(y, y^{\prime}, \frac{\Delta p}{p}\right)$, or, when solving misalignment problems on the column vectors $\left(x, x^{\prime}, 1\right)$ and $\left(y, y^{\prime}, 1\right)$. The code w\$ll construct 
other matrices representing assemblages of elements by means of the usual matrix operations. These matrices may be expressed in terms of the betatron function, which describe the betatron oscillation, and in terms of the eigenvector with unit eigenvalue which is the closed orbit if the matrix describes the whole accelerator, or a repeat period of the accelerator.

The code may be used to obtain the matrix product of all elements comprising the accelerator (or a repeat length thereof), to cycle that product with similarity transformation to obtain the one-turn transfer matrices starting from each of the constituent elements, and then to calculate the betatron function of these matrices. As a result, one may obtain linear orbit information at every point around the ring. Certain design problems for alternating gradient symchrotrons may be solved, such as the adfustment of an FDDF repeat length (or cell) to produce a specified phase advance, or the design of long straight sections.

Particle orbits may be traced through the synchrotron or through any part of it. For this purpose, the synchrotron may include non-linear elements.

7. MAGOP 22

This program calculates the orbit of a charged particle in median plane of a symetric magnetic field, and it determines first-order horizontal and vertical transfer matrices associated with the orbit.

MAGOP solves the vector equation of a changed particle moving in a static magnetic field defined by Eq. (1) 


$$
\frac{d^{2} \vec{r}}{d s^{2}} \quad \frac{g}{p} \frac{d \bar{r}}{d s} \times \vec{B}
$$

where $r$ is the position vector of the particle, $s$ is the arc length, $q$ is the particle change, $p$ the momentum and $B$ is the megnetic field.

The equations of motion of particles near the central orbit in the horizontal and vertical sections are taken to be

$$
\frac{d^{2} n}{d s^{2}}=-\left[\frac{g}{p} \frac{d B_{y}}{d n}+\left(\frac{q}{p} B_{z}\right)\right] n+\frac{g}{p} B_{z} \frac{\delta p}{p}
$$

and

$$
\frac{d^{2} \sigma}{d s^{2}} \frac{g}{p} \frac{\partial B}{d n} \sigma
$$

where $\mathrm{n}$ is the displacement of the deviating orbit in the median plane measured positively in the direction of the vector $\hat{k} \times \frac{d \vec{r}}{d s}$, $p$ and $\delta p$ are the momentum of the deviating orbit in the median plane, and $\sigma$ is the displacement of the deviating orbit in the vertical direction measured from the median plane.

One of two sets of transfer matrices is calculated depending on the sign chosen for the arc lengths. For a positive, $s$, the forward transfer matrices, corresponding to transformation from the initial to the final point, are calculated. For a negative $s$, the reverse transfer matrix is calculated. 


\section{Computer Programs for \\ Circuit Design}

1. CIRANB $^{24}$

This is a computer program use 1 to perform AC stendy-state and transient analysis of linear networks. The eircuit schematic and analysis options are described to the program by means of the CIRANB problem-description language, which is a format-free routine allowing the user to describe his circuit analysis problem in terms familiar to circuit designers.

It is a modified version of a linear circuit-analysis program written at the University of Illinois under the direction of D. A. Calahan. Some of the features of the program are:

1. Relatively free-format input description

2. Library of Input waveforms for use in transient analjsis

3. Capability of changing the analysis options and of altering the values of any arbitrary number of components for varlational analysis without redefining the complete problem.

4. Capability of adding or deleting components from the clrcuit for variatlonal anelysis without redefining the complete problem.

5. Writing of a plot tape for use by an adaitional plotting program, which can superimpose related curves from variationai analysis on the same graph frame. 
2. CIRCUS $^{25,26}$

This is a time response analysis program for electronic circuits. It is most suitable for analysing circuits where nonlinearities are essential for proper operation. All the present day junction devices are suitably represented by standard, accurate models.

It is the fourth generation of Branin's TAPl and the direct descendent of Malmberg et al's NET-1 ${ }^{2}$. Some of the main characteristics of this program are:

1. Flexible input format with minimal restrictions

2. Any current, voltage and power dissipation can be printed and plotted as output.

3. Junction device prarmeters can be stored in a library tape and be used when called. At present there are about 200 transistors and 20 diodes available.

4. Except for ohmlc resistences of the junction devices, all parameters cen be varied one at a time or all at once.

CIRCUS adopts the "state space" approach rather than the traditional nodal or loop formulation. The progran first converts the junction devices into their equivalent circult models with conventional $R, I, C$ and voltage or current generation. Then the topology of the circuit is translated into the incidence matrix of zeros and ones, with a " 1 " representing a branch connecting two nodes and a " 0 " if there is no branch connecting the two nodes. After this a "normal free" is p-cked to insure that the equations obtained later are all linearly independent. From the 
"normal free" and the incidence matrix, $\eta$ first-order differential equation can be written of the form

$$
[\dot{X}]=[\mathrm{Z}][\mathrm{X}]+[\mathrm{U}]+[\hat{\mathrm{S}}][\dot{U}]-[\mathrm{I}]
$$

where $[\mathrm{x}]=$ Independent branch currents and node voltage vector (these are state variables)

$[z]=R, I, C$ matrix

$[U]=$ Indepenlent current and voltage sources vector

[S] = Matrix for independent voltages and current sources

[S] = Derivative matrix for iLdependent voltage and current sources.

Dot implies first derivative.

Eq. I involves only first derivatives, and it is therefore relatively easy to solve. CIRCUS makes use of Pope's exponential numerical integration scheme which lessens the severity of the incompatibility problem between the circuit time constant and integration step size in cases where the latter becowes too large.

3. TERMI ${ }^{27}$

This is a wirelist program, written to provide a convenient way of recording all pin-to-gin connections and to generate printouts of the connections arranged in various tyays.

TERMI can be thought of as an interface between the designer of the circuits in a bin and the people who wire and debug it. Input essentially corisists of cards containing a function name, followed by a number of pins which are to be connected together to perform the function. 
Output consists of three lists. The first two are pin lists one arranged alphabetically in order of function, the other by order of pin-number. These two provide cross reference during debugging for tracing the wiring of a particular function or pin. The third list consists of pin-pairs, arranged by color. This list is intended for use during the wiring of the bin.

Colors may be assigned to particular function names so the color wili appear next to all pins of this function in the lists. Also, the pin-connection cards can specify connections to be made from a function to an external appliance (external in the sense that it is not located on a card connector) such as cable-plug, light, or switch. These external contacts may also be connected to each other.

3 zause other versions of the program are used for taper-pin connectors, a basjc assumption is that no more than two wires will be connected to any one pin. This might appear to impose excessive restristions in the connection routing for wire-wrap, where 3 or 4 connections can be made to a pin, but in prectice it is seldom much more complex. Also, the wiring density is kept lower, permitting easier use of scope probes. Each set of pins and external connections to be connected together must be giver a unique function name. This permits pins on several different IBM cards to be interconnected without the necessity of placing the cards together in the input deck. If no function name is given, the program w1ll make up one. 
4. BIAS $^{28}$

This program solves for the DC node-to-datum voltages and transistor operating points of circuits containing resistors, hipolar (NPN or PNP) transistors, current sourcss and grounded voltage sources. The nonlinear model ised for bipolar transistors is equivalent to that of Ebers and Mall. Additionally, i: requested, BIAS will calculate the smell sigral incremental input resistance and voltage gain for a specified part description. Analysis may be repeated for several values of an DC source and/or for several different temperatures.

The program is limited by the following topological restrictions

a) 40 dependent nodes + datum node

b) 40 resistors

c) 30 bipolar transistors (NPN or PNP)

d) 20 current sources

e) 20 grounded voltage sources

5. CORNAF ${ }^{29}$

The purpose of this program is to obtain the state equations and desired transfer functions, together with frequency and time responses of an $\mathrm{N}$-part linear active network. The program provides plots of frequency and time response as directed, by an easy to use format free language.

The linear anilysis performed by CORNAP is restrlcted to networks whose dimensions do not exceed 24 independent nodes and 64 uranches. The time invarient lumped linear elements of the network may be of the following types: 
1. Ordinary two-terminal passive circult elements $(R, L, C)$

2. Mutuel inductance and capecitance

3. The four two-terminal controlled sources (voltage/currentcontrolled voltage/current sources)

Two-port active and nonrectprocal elerents such as negative1mpedance converters, ideel transformers and gyrators can be made up of the one-part elements described above. Inputs are defined by attaching Independent voltuge and current sources to the network. The topological description of the network is given in terms of cards accompained by a few control cards dictating the quantities to be salculated and/or plotted. The output consists of a listing of the Input date and a listing of the poles and zeros and the given constants of the transfer function desired. The state and output-state matrices may be 1igted. Time and frequency response may be computed and the tabulated results plotted. 
6. PUZZLE 30

This program is designed to provide solutions to the printed circuit card design problem. It is capable of destignias twosided boards with a variety of components, and aultiplieity of interconnections (from 1 to 550). PUZZLE allows the user to code his beard dimenolons (with variable spacing on the connector tabs), thus yielding complete reprecentation of the rinished board.

Sore of the ismitutions of the progran are:

1. ise path leneths are not minimfed. The routing logic tends to prosuce short paths but no effort is and to asture that they are the shortest patha available.

2. Through hollze are not finlafied the snoothing routine teade to woduce the nuber of hoies carrytng lines bech Ind sorth through the surface the baters but the

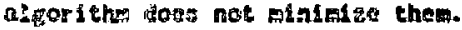

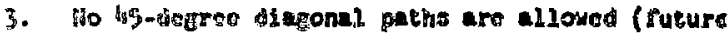
roditications did insorporate this fature).

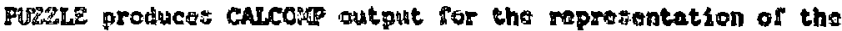
circuit thard, throe gipaph are producot for sach success ful routine. The rirat froph pieturs the board frow the parts side Wut skost woth aldes. The second graphe show all lines shat are to appers on the "parts 4 ido" of the bard. The third graph

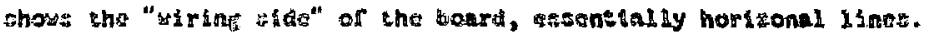


7. PICASSO

This is a general tnteractive graphles program for constructing a complicated date structure by the time-proven method of drawing pictures. The program can serve as "front-end" module for ans model-buildins program which interprets and analyoes a user-defined date structure.

In many applications the picture itself is the desired output, af in printed eircuit design layout or flow charting of procedursos and progruas, and for these, Prcasso is self-contained.

The philosophy of the progrea is to allow the user to create and edte diagrami, thich are treated as entities. The diagrans

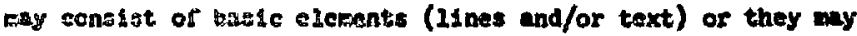
be tulit from othor (previousiy derined) diagrans es vell. The recusive nature of the data structure provides for the conAtruction of eadels based on user-derined components, such as Iayie diegrams (which cen serve as input to a wirewrap or sireult dowtgn and andysts progras), echanical englneering drautag, phys 1-al plant layout, optical systems desige, or elnost any problem where the frodsl is exter to draw than to encode in dedeal fors by thend. 


\section{APPENDIX I}

Bibllography

1. A. M. Winslon, Numerical Solution of the Quasilinear Polsson Equation In a Honuniform Trlangle Mesh. Journal of Corputational Physics, Vol. 1, No. 2, Nov. 1966.

2. J. S. Colonias, TRIM: A Magnetostatic Computer Program for the CDC 600 . Lakrence Radiation Latoratory, UCRL-18439, ALG. 1968.

3. J. Dorst. Private communication, Lawrence Radiation Laboratory, Berkeley.

4. S. J. Sackett, An Algorithm for the Numerical Solution of the Non-linear Magnetostatic-fleld Equation in Three Dimensions. UCID-3435, Lawrence Radiation Laboratory, Berkeley, Apr. 70.

5. N. A. Perkins, J. C. Brown, MAFCO-A Magnetic Fleld Code for Handling General Current Elements in Three Dimension, UCRL-7744 Rev. H, Nov. 1966.

6. \%. $H$. Garrett, An Elliptic Integral Computer Package for Magrets.c Fields, Forces, and Mutual Inductances of Axilsymetric Systems, and a Versitile Iine-tracking Routine. ORW-3575 Oak Ridge National Laboratory, April 1965.

7. C. D. Herinin, FORCE: A Computer Program for CaIculating Magnetic Forces Developed in Electromagnets, UCRL-14917, Mar. 1966.

B. R. Perin, S. Van der Meer. Frogram "MARE" for the Computation of Two Dimensional static Magnetic Fields, CERN 67-7, Mar. 1967.

9. S. Sackett, R. Healey, JASON - A digital Computer Program for the Numericaj, Solution of the Liaear Poisson Equation $(k \quad 0)+=0$ UCLAL-18721, Lairence Roriftion Laboratory, Berkeley, Feb. 1969. 
10. S. K. Howry, C. H. Moore, H. S. Butler, "TRAYSPORT". A Computer Program for Designing Beam Transport System. SLAC Internal Report. (1963).

11. A. C. Paul, Private comunication, Lawrence Radiation Laboratory, Berkeley.

12. T. J. Devlin, OPIIK: An IBM 709 Computer Program for the Optice of High-Energy Particle Beam. UCRL-9727, Lawrence Radiation Laboratory, Sept. 1961.

1j. R. Chaffee, Private cormunication, Lawrence Radiation Laboratory, Berkeley.

14. J. W. Gardner, D. Whiteside, FORRAN Version of TRAMP, NIRL/M/4l, Rutherford High Energy Laboratory, Jan. 1963.

15. D. Kane, Frivate communication, Lawrence Radiation Leboratory, Berkeley.

16. E. R. Close, CYDE: A Collection of FORTAA II Cyclotron Development Codes for Us6 on the IBM 7094, UCID-2869, Lawrence Radiation Laboretory, Berkeley.

17. M. M. Gordon, T. A. Welton, Computation Methods for AVF Cyclotron Design Studies, ORRL-2765, Oak Ridge NatIonal Laboratory, Sert. 1959.

18. A. C. PQul, "TRAJECTORY" Orb1t and Ion Optic Katrix for the 184 in. Cyclotron, WGL 19407, Lakrence Radiation Laboratory, Berieley, Nov. 1967.

19. E. R. Close, SOTM: A Peogram to Generate F1rst and Second Order Matrix Elements by Tracking Changed Particles in a Specified Magnet1c Field, UCRI-19823, Lawrence Radiation Laboratory, Berkeley, May 1970. 
20. E. R. Close, Generation of First and Second Order Transformation Elements from a Given Magnetic Field, UCRL-19812, Lawrence Radiation Leboratory, Berkeley, April 1970.

21. M. Reiser, J. Kopf, "Electrolytic Tank Facility and Cosputer Program for Central Region Studies for the MSU Cyclotron MSUCP-19 Michigan Sțate University, May 1964.

22. V. Brady, Private comminication, Lawrence Radiation Laboratory, Berkeley.

23. A. A. Gerren, J. W. Eusebio, swiH: A Computer System for Synchrotron Design and Orbit Anslysis, JCID-10153, Laurence Radiation Laboratory, Berkeley, Apr. 1965.

24. G. S. Tooi, J. E. Katz, CIRANB: Linear-Circuit Analysio ProgramUser's Manual, UCRL-18185, Lawrence Radiation Laboratory, Berkeley, Apr. 1968.

25. E. K. Chen, A. Comprehensive Study of CIRCUS, UCKL-19329, Laurence Radiation Laboratory, Berkeley, June 1969.

26. E. K. Chen, H. Jackson, EIRCUS User's Manual, EE-1319, Lawrence Radiation Laboratory, Berkeley, June 1969.

27. B. Lafore, WIRELIST Program Operating Instructions: WBRII II: UCID 3287, Lawrence Radiation Laboratory, Oct. 1968.

28. S. Ratner, BTAS User's Manual EE-1345, L\&wrence Radiation Laboratory, Berkeley, Dec. 1969.

29. J. E. Katz, A Linear Analysis Program CORNAP, UCID-3411, Lawrence Radiation Laboratory, Berkeley, Nov. 1969. 
30. R. Zane, D. A. W. Harrell, Computed-Alded Design of PrintedCircuit Artwork, UCRL-18172, Lawrence Radiation Laboratory Apr. 1968.

31. 
35

APPENDIX II

PROGRAM SUMAARY FORM

Date May 10,1971

Title of Program

TRIM

I.D. Sumber EMM - 1

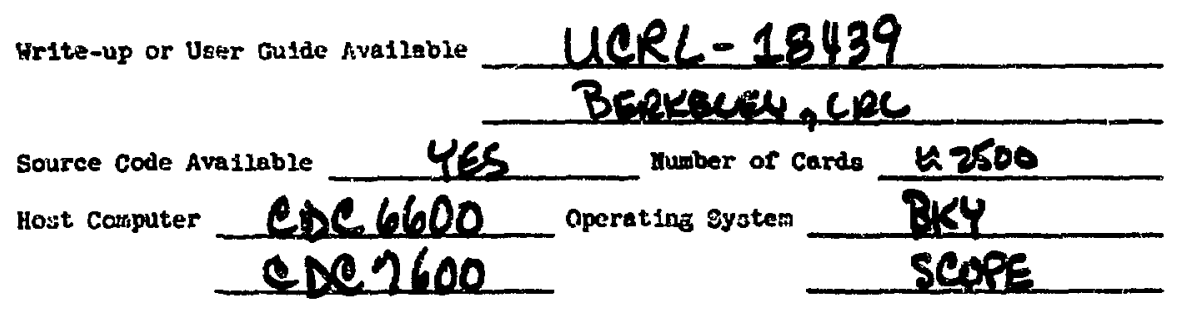

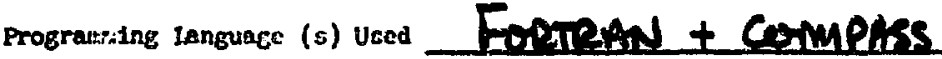

Menory Requirements

Averoge Running Tise $15-20$ MiN, thuber of mgnetic pupes Required 1

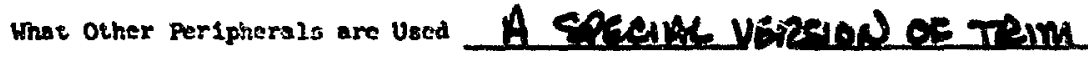

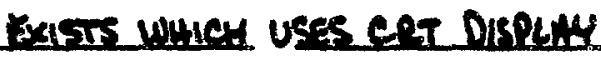
andinge.s.

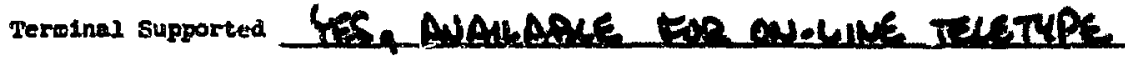

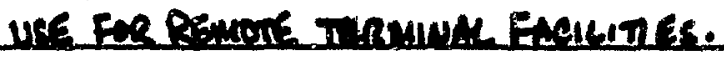

Reutrietione

Memory requirenents diand on the Mch sire used. The above at $156 \mathrm{~K}_{\mathrm{g}}$ is maxnmen sace of 4000 poinds. 
30

FROGRAM GUMARY TORM

Date 10,1911

Title of Program

LINDA

I.D. Number

EM-2

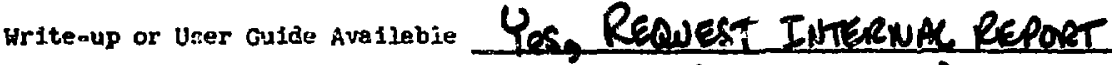
(UNPURHSHED)

source code avallable _ 168 Number of caràe $y(1,00)$

Host computer $\quad$ De 1.600 Operating Syster

CDC 1600

Programal Ianguage (s) Used

Forrenan ons

Momory Regulfenents

$210 k$ Program Ovarlata No

Average Runnits Time LD 4 MiN. Numer of Mgnetic Tapes Required 2

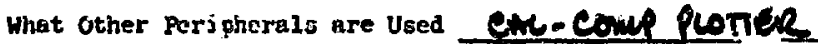

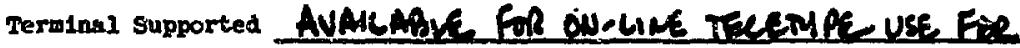

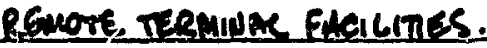

Restrictions

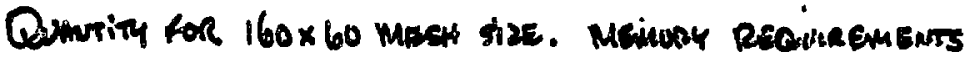
Depent on mesh isire sewectad. 
37

PROGRAM SUMMARY FORM

Date 49412,1971

Title of Program The

T.D. Number EM -3

Write-up or User Guide Ava1].able

No

Source Code Available

No Number of carats 41000 Host computer $C D C 7600$ Operating System BK scope.

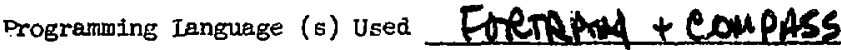

Memory Requirements $120 \%$ Program overlaid $\mathrm{NO}$ Average Running Time 1,2 Hes Number of Magnetic Tapes Required. What other Peripherals are Used NONE

Terminal supported No

Restrictions NoT OPERATONA THS TME, FRESENTLY BENG TESTED.

* Redineza for a $31 \times 31 \times 21$ mesh.

Memoriser requirements vary, depending on mesh Sleeted. 
38

PROGRAM SUMMARY FORM

Date MAM 10,1971

Title of Program

MAFCO

I.D. Number EM -4

Writeup or JEer Guide Available

LCRL-7744 CRC.BERKELYY

Source Code Available Number of cards $\propto 1200$

Host Computer exc 6600 Operating System code 2600

Programming Language (s) Used FortrAn

Memory Requirements

$67 \mathrm{~K}$

Program Overlaid

No

Average Running Time $1-3$ MiN. Number of Magnetic Tapes Required NoNE

What other Peripherals are Used None

Terminal supported AJANABUE FOQ ON-LINE. TEETMUE USE FUR Remote TERMINAE facilities.

Restrictions 
39

PROGRAM SUMMARY FORM

Date Per 15,1911

Title of Program

Corks

I.D. Number

EM-5

write-up or user Guide Available ORNL-3575 OAK Rinse shover

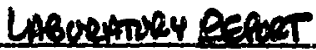

Source Code Available L_ LS. Number of cards $\sim 200$

Host Computer cDc 6600 $C D, 7600$

Puss scare.

Programming Language (s) Used Operating System

Memory Requirements Program Overlaid No Average Running Time $\leq 60$ see. Number of Magnetic Tapes Required NONE What Other Peripherals are Useà NONE

Terminal supported Ad HLPRE FOR online TECEMPE USE fR R PEMOTE TERMInAL freintses.

Restrictions 
40

PROGRAM SUIARRY FORM

Date SUN E 1,197t

Title of Program

I.D. Number Ency

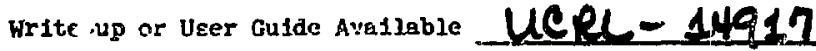
CAREer

Source Code Available Yes Number of cards 42000 Host computer exc 66000 Operating system CoDe 1600

Programing Language (s) Used Timon.

Memory Requirements SOKe Program Overlaid No

Average Running Time 460 sal Number of Magnet:- Tapes Required

What Other Peripherals are Used None.

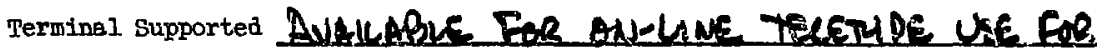
mote. TERMINAC FNALITIES.

Restrictions 
41

PROGRAM SUNSARY FORM

Date MKY29,1911

Tit.1: or Progren . MAREC 1.1.) kirmuler $-E M-8$

wrste-up or uner Gusde Ares:abis C.E.R.N. 67-7

Source Code Avallatle

Hosi Computer coe 6600 CDC 760

Programing Lenguage (s) Used

Memory Requirements
Number of cards oprieting system

BYY

seupe

Average funning Time S-10 MW. Number of Magnetic Tapes Required

What other Fer1pherals are Used NONG

Terminel Supported

$\mu_{0}$

Restrictions

USER REQUIREA DWWRE HS DWN QUTPUT ROUTNE(S)

TD PRINT BX, BY COMPONENTS OF. THE MACNERTC FIELD.

* time for u 3000 points in iron. 
PROGRAM SUMMARY FORM

Date IUNE 10,1991

ingle or Program

JASON I.D. Number EM-9

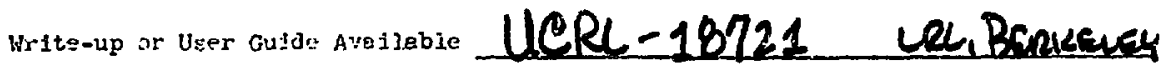

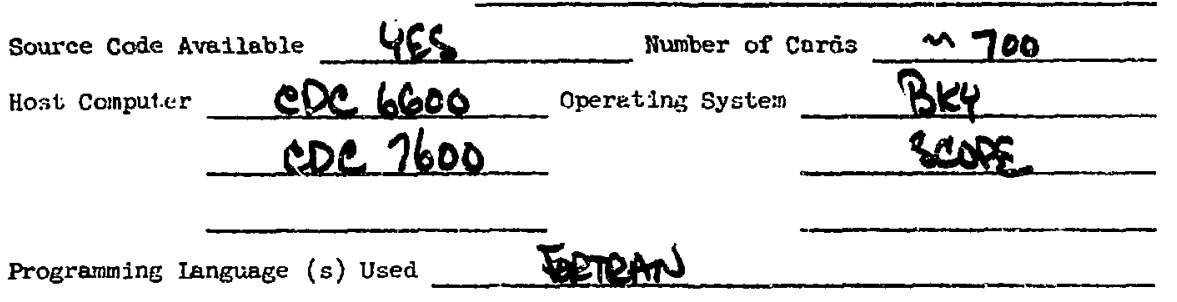

Memory Requirements

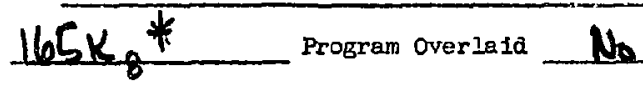

Average Running Time 3-5 Mid. Number of Magnetic Tapes Required 1 What other Peripherals are used CAx-CAwP PLotTer

Terminal Supported He

Restrtattons

* For a zoo point mesh. 
43

PROGRAM SUIHARY FORM

Date MAY 10,1971

Title of Program TRNSPORT I.D. Number BI 1

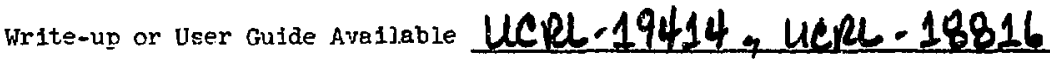
Q14 BePLeney Source Code Available 455 Number of cards Host computer CDCla600 Operating System BKS CDC 1600 SCOPE

Programming Language (s) Used Fran

Memory Requirements $60-100 \mathrm{~K} 0^{* / 3}$ Program over laid No enrage Running Time Number of Magnetic Tapes Required Lavs What other Peripherals are Used C QR Disflit Cougoce) TELEMPE $\} \frac{\text { INTERACNNG VERSION }}{\text { ONLY }}$ Terminal supported A SPECIAL VERSION UE THE CODE EXISTS FR THE ON-UINE TELERTPE USE FR R PEMDTE FAtaLities. ANOTHER VERSION BLOWS GRAPTIC-MSPLAY INTERACTION.

Restrictions

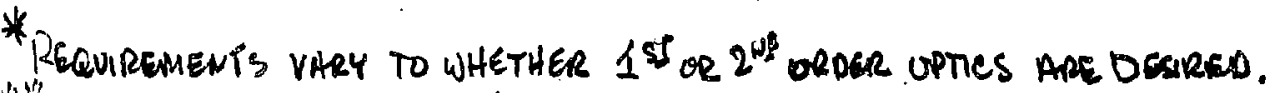
TMEE ALSO VARRES FOR DIFFERENT VERSAOWS, 1-10 win. 
Date JUWE 10-1971

Title of Proisram I.D. Number

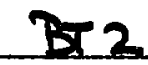

Write-up or User Guide Avallable UCRL- 9127

LRE, BERKENAY

Source Code Avalleble Number of carás u 4000

Host Computer

esc 6600 Operating Systein

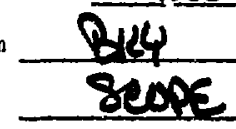

Programing Lenguage

(s) Used

Foetrans

Memory Requirements

SOK

Program Overlaid

No

Average Running Time $1-2$ Min. Number of Magnetic Tapes Required Noys What other Peripherals are Used CAL Disflay CaN zocs. TELETPS

Terminal supported THERE is A SPEEIAL GRAPHL-INTERACTIDal VERSSOW.

Restrictions 
45

PROGRAM SUMmARY FORM

bate IUNE 3,197

site of Program

TRAMP I.D. Number

Frite-up or User sulde Avasiable NI RL/m/41 JWJ. 1413

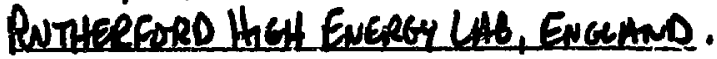

Source Code Available

Host Computer Number of Cards Yes 0006600 Operating System $\operatorname{coc} 7600$

Programing Language (s) Used

Memory Requirements

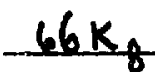
Program Overlaid No Average Running Time $1-2$ id. Number of Magnetic Tapes Required No ll, What Other Peripherals are Used None.

Terminal Supported No.

Restrictions 
PROUIAN GUKHARY FORA

Date Sughe 20,1951

Title of Program

CYDE.

I.D. Number

Q-1

write-up or ters Gulde Ave1jeble UCID -2869

CRL, B60ucuer

Source Code Avaslable Number or cardo

Host Computer exc 6100 opereting syotem exc 7600

Frograming Lenguage (s) Used

Ala Picgram Overlaid No

Memory Requirements Miv. Number of Negnetic Tapes Required Average Running Time Nouse

What Other Peripherals are Used

Terminal supported?

Restrictions

* Under dengcopuent for on-lime tecetupe ve for renore fallities. 
Title of Profram

$\operatorname{GeC} 3 D$

I.D. Number

$\theta-2$

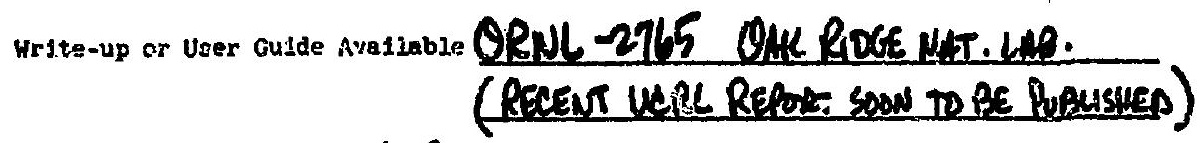

Source Code Availuble 435 Number of Cards

Host Computer

edC 6600 operating system

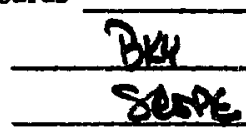
$\operatorname{coc} 2600$

Progransoling Ianguage (s) Used Fempins

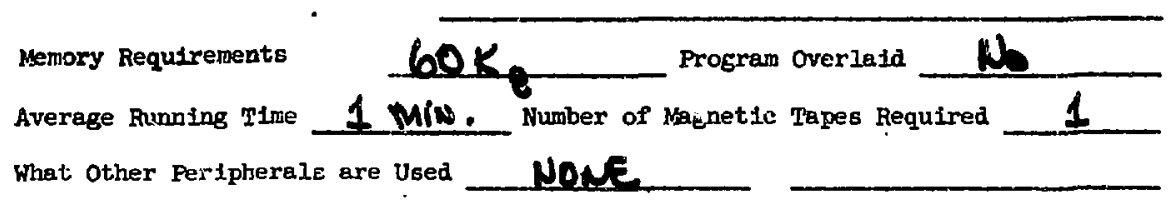

Terminal Supported No 
48

PROGRAM SUMMARY FORM

Date MAY 20, 1971

Tit :e of Program

TRAJECTORY

I.D. Number $\theta-3$

writeup or User GuIde Ave11able UCRL -19407 LRL, BeRKELEy

Source Code Available Yes Number of Cards

Hast Computer $\operatorname{cose} 6600$ Operating System enc 1600 BuY Y Saps.

Programing Language (s) Used Fortran

Memory Requirements 505 Program overlaid No Average Running Time $1-2$ minN. Number of Magnetic Tapes Required Nous What Other Peripherals are Used None

Terminal supported No

Restrictions

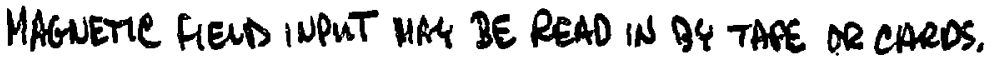




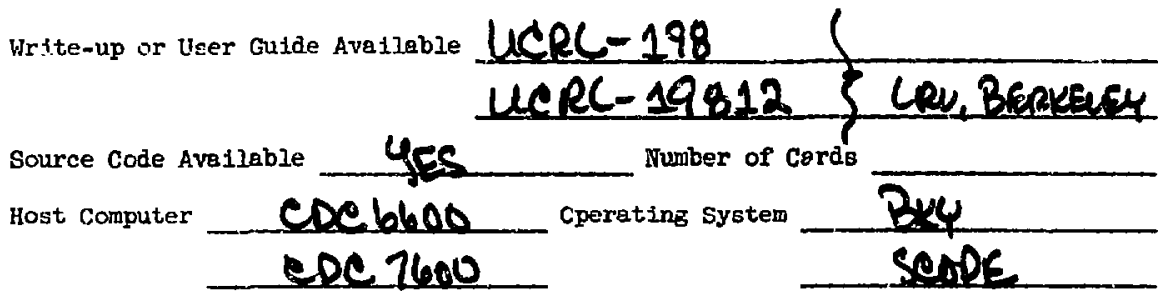

Programing Language (s) Used

Eorman

Memory Requirements Average Funning Time What Otiner Pertpherals are Used Program Overlaia

No $3,4 \mathrm{Nin}$. Number of Nagnetic Tapes Required Nove.

Terminal supportea No Restrictions 
Write-up or User Guide avaliable ReQUfe, COPY From LiRL COMPUTER CENTER, BERKELER.

Source Code Available

YES Number of Cards

untooo

Host Computer

ede 6600

\section{coc76od}

scope

Programing Language $(s)$ Used

Fortanas

Memory Requirements

S5K_____ Program Overlaid

No

Average Running Time

1 Min. Number of Magnetic Tapes Required

None What other Peripherals are Used CAL-COMP ROTTER

Terminal Supported

No

Reetrictions 
51

IROGRAM SUMMARY FORM

Date MAY 10,1971

Title of Frogram SYNEH I.D. Number $\theta-6$

write-up or user Gulde Available UCID-10153, LRL BERKkLEY

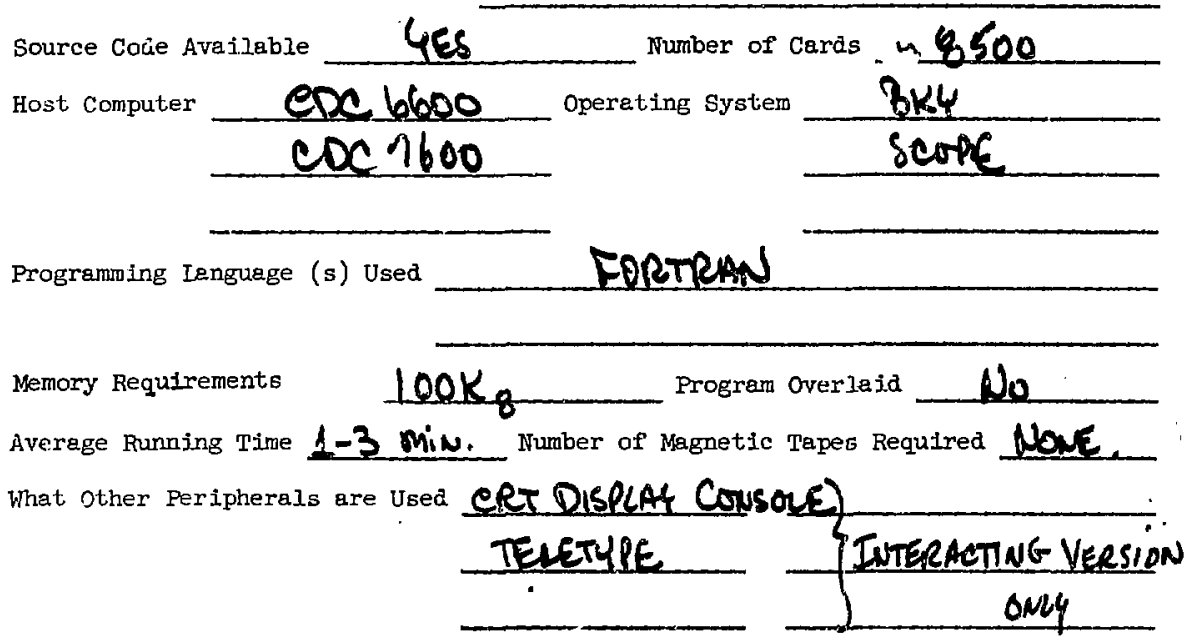

Terminal supported IHERE IS AN INTERACTING GRAPH:C-DISPLAY VERSION.

Restrictions 
52

PROGRAM SUMMARY FORM

Date MAF 14.1971

Title of Program MAG QP

I.D. Number

Q-7

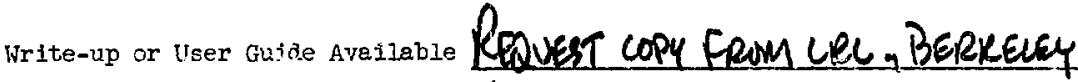
Computer center.

Source Code Available Number of cards 220

Host Computer

ede 6600 Operating system enc 7600

Progranining Language (s) Used

Memory Requirements SoL. Program Overlaid No Average Running rime 1 miN Number of Magnetic Tapes Required What Other Peripherals are Used pone

Terminal Supported No

Restrictions

* Magnetic field input caus be read in by. tape urchros. 
Title of Program CIRANB I.D. Number $C A D-1$

Write-up or User Gutde available UARL-18185 \& LRL Berkeley Source code Available YES Number of Cards Host Computer DDC 6600 Operating System BKK

Programmine Larguage (s) Used Foenows Memory Requirements SOKg_ Program Overlaid _. No Average kunning T:Ime $2-3$ min. Number of Magnetic Tapes Requirea HewE What other Peripherals are Used CPL-comp PlotTER Terminel supported No. Restrictions 
54

PROGRAM SUMMARY FORM

Date Sulu 20,1971

Title of Program

CIRCUS

I.D. Number

CAD .2

Write-up or user guide Available LCRL-19329, GRe BERKELEY EE-1319, CRU BERKELEY ENGIN:

Source Code Available Number of Cards

Host Computer eDe. 6600 Operating System

Programming Language (s) Used

Memory Requirements

$127 \mathrm{Ko}$

Program Overlaid

No

Average Running Time 1 Mil.

Number of Magnetic Tapes Required Now th

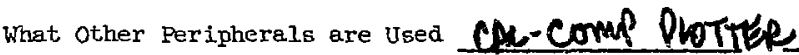

Terminal Supported

No

Restrictions

AN INPUT TAPE MAS BE USES TO PROVIDE CODE. WITH A TRIASISTEZ MOOSE GARRY. 
Title of Program

write-up or veer cusae Available UCID. 3287 LRC, BERkELEY

EE-1278** LR, BERKELEY

Source Code Available $4 E S$ Number of cards

Host Computer CDC6600 Operating system

Date Juer 20, 1971

Bky

Programming Language (s) Used

Fortrint

Memory Requirements

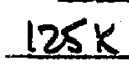
Program Overlaid

No

Average Running Time

20

MiN.

Number of Magnetic Tapes Required

Nowe

What Other Peripherels are Used

None

Terminel Supported

No

Restrictions

$\frac{1}{4}$

THHS CODE WIU SON BE REPLACED BY PROGRAM WIRELIST. ** Encruegring Dept. 
56

PROGRAM SUMMARY FORM

Title of Program BIAS

Date fury 21, 1971 I.D. Number $C A D-4$

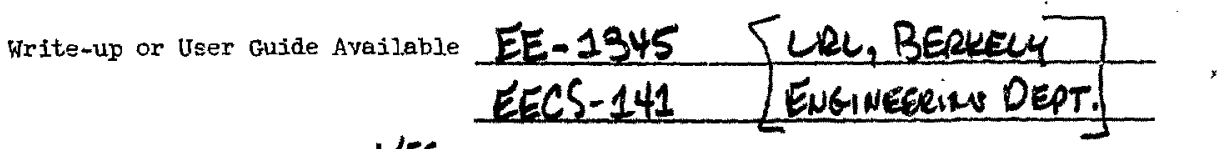

source Code Available

Host Computer CDe6600 Number of Cards Operating System BKS

Programming Language (s) Used

Memory Requirements $100 \mathrm{~K}$ Program Overlaid No Average Funding Time 1-3 MIN. Number of Magnetic trapes Required NONE What other Peripherals are Used Nome

Terminal supported No

Restrictions 
Title of Program CQRNAP T.D. Number PAD-5

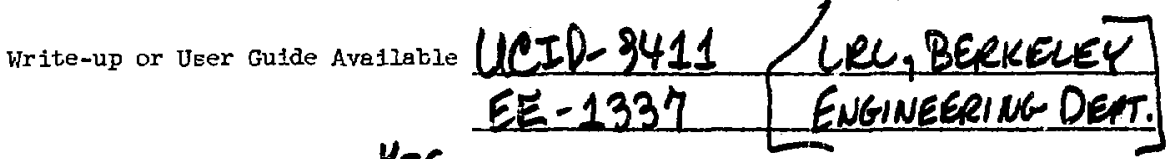
Source Code Available 4 ES_____ Number of Cards Host Computer $\mathrm{CDC6} 600$ operating system $\mathrm{DKY}$ Programming Language (s) used___ Foetrend Memory Requirements $101 \mathrm{~K}_{\mathrm{O}}$ Program Overla1a Average Running Time 1-3 Mil Number of Magn :Ic Tapes Required What other Peripherals are Used CAL-Comp Plortegs

Terminal supported No 
PROGRAM SUMMARY FORM

Date Sulk 25,1971

Title of Program

Puzzle

I.D. Number $C A D-6$

Write-up or User Guide Available UCRL-18172 CR L BERKEEVEr

Source Code Available 425 Number of Cards

Host Computer $\mathrm{CDO}, 6 \mathrm{COO}$

Operating system Bks

Programming Language (s) Used

Memory Requirements $125 K_{8}$ Program Overlaid No

Average Running Time 10-1S SAA. Number of Magnetic Tapes Required

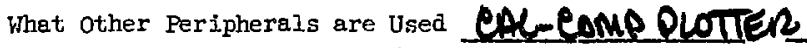

Terminal supported No

Restrictions 
Title of Progrem

PICASSO I.D. Number

CAD-7

Write-up or User Guide Aveilable (LCLPL PAPER PENDING)

Source Code Aveslable LES Number of Cards Host Computer coc6600 Operating System

BK4 SCAPE

Programming Language (s) Used

Foprens

Bump

Memory Requiraments

$50 \mathrm{~g}$

No

Average Runnting Time UAPAARUK Number of Magnetic Tapes Required

1 What Other Peripherals are Used.

MODEL 52 PISPAAY CONSOLE TELPMPE

Terminal supported

No

Restrictions 